\title{
Saberes educativos mapuches: un análisis desde la perspectiva de los kimches*
}

\author{
Daniel Quilaqueo R.** \\ Segundo Quintriqueo M."
}

\begin{abstract}
Resumen: Este artículo aborda la acción educativa mapuche kimeltuwün como aporte a la solución del problema que implica la contextualización de los contenidos que se enseñan en la escuela a niños y niñas de origen mapuche que inician su proceso de escolarización. Se trata de saberes vernáculos que se centran principalmente en contenidos actitudinales relacionados con la memoria social mapuche. Se revelan conceptos y métodos educativos que utilizan los kimches (sabios portadores del conocimiento social y cultural) para formar a niños, niñas y jóvenes en el contexto de la familia y comunidad. Para ello, los sabios recurren a la lógica sociocultural sobre la cual se fundamentan sus saberes educativos. Los resultados muestran la vigencia de categorías de conocimientos educativos mapuches para la formación de las personas.
\end{abstract}

Palabras clave: saberes educativos, saber mapuche kimün, sabio o kimche.

\section{Mapuche educational knowledges: an analysis from the perspective of the Kimches}

\begin{abstract}
This article presents the kimeltuwün Mapuche educational action to contribute to the solution of the problem involving the contextualization of school contents to teach Mapuche children who begin their schooling process. It is about vernacular knowledge mainly focused on attitudinal contents related to the Mapuche social memory. Educational methods and concepts used by kimches (wise men bearing social and cultural knowledge) to educate children and young people in the family and community context are revealed. To do this, the wise men look upon the sociocultural logic on which their educational knowledge is based. The results show the validity of Mapuche educational knowledge categories in teaching people.
\end{abstract} or kimche.

Key words: educational knowledge, kimün Mapuche knowledge, wise man

\footnotetext{
* Este artículo es parte de los resultados del Proyecto Fondecyt N 1085293 "Racionalidad del método educativo mapuche desde la memoria social de kimches: fundamentos para una educación intercultural” (Quilaqueo, Quintriqueo, 2008-2010; investigadores del Núcleo Iniciativa Científica Milenio: Centro de Investigación en Educación en Contexto Indígena e Intercultural CIECII).

** Universidad Católica de Temuco, Temuco, Chile. Email: dquilaq@uct.cl

*** Universidad Católica de Temuco, Temuco, Chile. Email: squintri@uct.cl
} 


\section{Saberes educativos mapuches: uma análise sob a perspectiva dos kimches}

Resumo: Este artigo aborda a ação educativa mapuche kimeltuwün como uma contribuição para a solução do problema que envolve a contextualização dos conteúdos ensinados na escola às crianças de origem mapuche que iniciam seu processo de escolarização. Trata-se de saberes vernáculos centrados principalmente em conteúdos atitudinais relacionados com a memória social Mapuche. São revelados conceitos e métodos educativos que utilizam os kimches (sábios portadores do conhecimento social e cultural) para formar meninos, meninas e jovens no contexto da família e da comunidade. Para isso, os sábios recorrem à lógica sociocultural sobre a qual se fundamentam seus saberes educativos. Os resultados mostram a vigência de categorias de conhecimentos educativos mapuches para a formação das pessoas. kimche.

Palavras-chave: saberes educativos, saber mapuche kimün, sabio ou

Recibido: 29.09.2009

Aceptado: 02.05.2010

$* * *$

\section{Introducción}

Los saberes educativos mapuches vitales, utilizados por las familias en la formación de sus hijos, siguen vigentes entre las familias de comunidades de la Araucanía. Dichos saberes se sustentan en métodos de formación de personas y principios propios que los regulan. Son saberes vitales que se relacionan con la educación mapuche y que es necesario retomar para el diálogo cultural entre alumnos de origen mapuche y no mapuche. La conceptualización de estos saberes, que aquí se presenta, es el resultado de los datos recopilados de manera sistemática y se analiza a partir de la Teoría Fundamentada de Strauss y Corbin (2002). Desde la perspectiva mapuche, se recurre a su lógica cultural sustentada en valores de la persona y de quienes asumen la calidad de kimche (Quilaqueo, 2006). Esta condición implica poseer conocimientos socioculturales mapuches y no mapuches para desarrollar un discurso ${ }^{1}$ educativo.

La problemática que se investiga plantea que en las comunidades y en los nuevos contextos de vida ${ }^{2}$ mapuche es posible reconocer a los kimches como educadores tradicionales expertos en conocimientos culturales y so-

\footnotetext{
${ }^{1}$ El discurso se entiende como un complejo de tres elementos interrelacionados e interdependientes entre sí: el texto y sus elementos constitutivos, la práctica discursiva que los hablantes realizan a través de los textos y la práctica social donde tanto práctica discursiva como texto son modelados ideológicamente (Van Dijk, 1994; Fairclough, 1995).

${ }^{2}$ Se denomina nuevo contexto de vida mapuche al asentamiento de éste en el medio urbano como efecto de la emigración y en las dos últimas décadas en el medio rural como efecto del programa de restitución de tierras, por parte del Estado, en nuevas localidades fuera del la comunidad familiar.
} 
ciales que fundamentan los métodos educativos que utilizan. Estos conocimientos son enseñados mediante una acción educativa denominada kimeltuwün (Quilaqueo, Quintriqueo, 2008). Esta acción educativa se refiere a la contextualización y vinculación de los contenidos de aprendizaje con aspectos culturales del medio natural y del medio social en los que se ha desarrollado la familia y su comunidad. Sin embargo, la acción educativa kimeltuwün ha encontrado dificultades cuando los niños y niñas inician su escolarización, dada la descontextualización de los contenidos que se enseñan en la escuela. La hipótesis de trabajo que aquí se plantea es que los kimches conocen los fundamentos, valores y finalidades educativas de los saberes y conocimientos socioculturales mapuches, y han sido los depositarios de saberes específicos sobre la formación de personas y de los oficios. Esta formación se realiza mediante el discurso oral, en mapunzugun (lengua mapuche), desde una lógica de construcción de conocimiento que permite dar significados a los elementos culturales mapuches y no mapuches, a través de una modalidad de apropiación cultural (Bonfil-Batalla, 1987; Quilaqueo, 2005, 2007).

El problema y la hipótesis se indagan desde una perspectiva sociológica, intercultural y pedagógica. Estos enfoques permiten identificar los saberes educativos en contextos de diversidad cultural (Molina, 2002). Además, admiten resultados de investigaciones empíricas sobre saberes indígenas y el saber del diálogo cultural en la definición de contenidos y finalidades educativas (MINEDUC, 2005). En síntesis, para indagar en el saber educativo mapuche, desde el rol de los kimches, se consideran contenidos conceptuales, procedimentales y actitudinales (Bixio, 1997; Quilaqueo et al., 2004), para develar lo que los kimches describen como saberes vitales que estimulan una educación mapuche.

\section{Marco teórico}

Los enfoques teóricos que sustentan este trabajo se apoyan en lógicas de estudios socioeducativos tanto mapuches ${ }^{3}$ como no mapuches, y a partir de un planteamiento sociológico que señala que no se puede comprender la vida social actual de los pueblos indígenas si no es poniendo al sujeto individual como elemento central donde la vida social implica obligaciones de las comunidades sobre los individuos que forman parte de ella (Bajoit, 2003). Esta perspectiva intenta explicar las conductas mapuches colectivas, el orden y el cambio en las comunidades a partir del conocimiento de los kimches. Los sujetos individuales, que a la vez son sujeto y objeto de sus relaciones sociales, buscan construir y realizar su identidad personal mediante intercambios que le permiten comprender la vida social. Como enfoque epistemológico, este estudio busca conocer los saberes edu-

\footnotetext{
${ }^{3}$ Estudios realizados por investigadores de la Facultad de Educación de la Universidad Católica de Temuco y, actualmente, investigadores del Centro de Investigación en Educación en Contexto Indígena e Intercultural (CIECII).
} 
cativos mapuches, apelando a la perspectiva teórica de indagar con los mismos individuos, considerándolos, simultáneamente, como objeto y sujeto de sus relaciones sociales, para explicar las conductas colectivas respecto de los saberes educativos desde la memoria social (Montesperelli, 2004), y como crítica epistemológica en la desigualdad de oportunidades (Van Haecht, 1999). En consecuencia, se trata de iniciar una explicación fundada en el paradigma de la identidad cultural (Bajoit, 2003).

Partiendo de una de las proposiciones de Bajoit, en la que plantea que "la vida social implica apremios del colectivo sobre los individuos que forman parte de éste” (2003: 31), los saberes educativos mapuches son vitales. La vitalidad de estos saberes se encuentra amenazada ya que no se han resuelto los problemas que implica la escolarización descontextualizada. Así, cada mapuche y no mapuche es instado por los kimches para que contribuya con una educación más contextualizada con el objeto de resolver el problema del desconocimiento de los saberes educativos mapuches en la escuela. Esta situación es tan importante que puede ser equivalente a dificultades de tipo económicas y sociales. El problema del desconocimiento, como dificultad, se refiere al concepto de relación con el saber que manejan los kimches, para describir la resistencia de quienes no aceptan otros saberes y conocimientos educativos.

En este trabajo, se recurre al concepto de relación con el saber propuesto por Beillerot (1998), ya que se refiere primero al reconocimiento de todos los saberes de los individuos, en particular, por parte de quienes se esfuerzan por eliminar al sujeto de la historia y, segundo, porque la relación con el saber puede entenderse como la del sujeto que preserva el aspecto práctico de la noción. Según Beillerot, el saber no se designa como saber sino como un vínculo de un sujeto con un objeto. Es un vínculo que permite abordar el develamiento de los saberes vernáculos en la formación de personas que está depositada en los kimches. De esta manera, los saberes forman parte de un sistema simbólico al que se añaden reglas de uso “...producidos en un contexto histórico y social; hacen referencia a culturas, expresan, muestran modos de socialización y de apropiación” Beillerot (1998: 23).

En investigaciones de Bradford (1976) y Bengoa (1987) en las que se refieren a la enseñanza que recibían los niños y las niñas, reconocen algunos aspectos de la educación mapuche. Entre ellos, Bradford alude a valores como el respeto y la capacidad de reconocimiento con el medio natural y social. Estos aspectos se resumen en la forma de enseñar, esto es sugiriendo, demostrando y animando al niño o niña a aprender. Por su parte, Bengoa, refiriéndose a las antiguas familias mapuches, señala que su educación consistía en ejercitar la memoria, el culto por los detalles, la precisión al describir las características de objetos y situaciones, donde el mapunzugun se caracterizaba por su riqueza descriptiva. El niño o niña era ejercitado en la descripción detallada de los cerros, de los animales, de las plantas y de los diversos elementos de la vida cotidiana, es decir, en las clasificaciones que lo iban formando desde los saberes y conoci- 
mientos de cada familia y comunidad. Estos conocimientos son los que históricamente han sido enseñados por personas reconocidas con la categoría social de kimche.

La condición de kimche ha sido definida en mapunzugun como sabio, docto y erudito (Augusta, 1903; Erize, 1960) y, desde el punto de vista socioeducativo, como sabios portadores del conocimiento educativo (Quilaqueo, 2005 y 2007). En efecto, el kimche, hoy día, puede definirse como la persona que posee conocimientos mapuches y no mapuches, cuyo objetivo es argumentar un discurso educativo (Quilaqueo, Quintriqueo, Catriquir y Llanquinao, 2004). Esto se puede constatar actualmente entre las distintas comunidades, ya que hay hombres y mujeres considerados kimches por sus conocimientos respecto de la educación mapuche (Alchao, Cariman, Ñanculef y Sáez, 2005). Los kimches conocen métodos de enseñanza para transmitir valores sobre los cuales se fundamentan los saberes mapuches (Quilaqueo, 2006), por ejemplo, respecto de la actitud de aprendizaje del niño o niña y respecto de la persona que asume las características de kimche cuando le corresponde enseñar (Quintriqueo, 2002; Cavières, et al., 2005). Los saberes que emplean se sustentan en la memoria social (Halbawch, 1970; Le Goff, 1991; Sabourin, 1997; Montesperelli, 2004), que entre los kimches se encuentra por segmentos que cada uno utiliza para enseñar. Además, se observa en el uso del weupin, que se define como confrontación de saberes para construir conocimientos. Estos conocimientos poseen características comunes como productos humanos externos al individuo y accesibles a los miembros de la comunidad, es decir, la memoria no es sólo un acervo de conocimientos interior a cada individuo, sino que se proyecta al exterior. Ésta se vuelve colectiva e intersubjetiva al ser compartida, ya que va más allá de su objetivación (Berger y Luckman, 2002; Montesperelli, 2004).

Dentro de la educación mapuche hay varios conceptos que se relacionan con la enseñanza y el aprendizaje. Con respecto al concepto kimün, éste se define en general como saber (Augusta, 1903; Erize, 1960; Catrileo, 1995; Quilaqueo y Quintriqueo, 2007), pero, además, se traduce como conocer, aprender, sentir y adivinar. La palabra kimün proviene de la raíz kim, que en su carácter de adjetivo significa sabido y entendido. Augusta, en su gramática señala que kim “...es la radical del verbo kimn” (1903: 138), que traduce como saber, aprender y sentir. Así, la ante sílaba kim porta, a lo menos, tres campos de significados. Uno de los significados se vincula con conocimiento y saber que, como sustantivo, significa sabiduría; un segundo campo semántico se vincula con lo educativo, que incluye el binomio enseñar y aprender; $y$, por último, tiene otro significado que se vincula con la idea de toma de conciencia. Desde las ciencias sociales, para Foucault, "un saber es aquello de lo que se puede hablar en una práctica discursiva...” (1997:306), mientras que para Lyotard (1994), refiriéndose al saber científico, remarca que éste no es todo el saber ya que siempre ha estado en excedencia, en competencia, en conflicto con otro tipo de saber para simplificar, este autor lo denomina saber narrativo. En tanto, Ogien, conceptualiza el saber como saber de sentido común y como conocimiento 
corriente, puesto que son “...dos nociones que la sociología emplea a menudo como si fueran análogas” (2007: 96).

Para referirse a la educación mapuche, se reconocen cuatro conceptos: yimümün, yewmewün, mümülkan y kimeltuwün ${ }^{4}$ (Augusta, 1903; Erice, 1960; Quilaqueo y Quintriqueo, 2007). Para el análisis y discusión de los resultados de este artículo, se considera el concepto kimeltuwün, que es definido como acción educativa que implica un proceso de aprendizaje y enseñanza entre dos personas que tienen intención de abordar un contenido de aprendizaje. Un ejemplo de lo anterior es el aprendizaje del parentesco mediante los conceptos de tuwün (ascendencia materna-territorial) y küpan (ascendencia paterna), que sigue la referencia de la memoria social, con una marcada vinculación con tiempo pasado (Quintriqueo, 2005; Quintriqueo y Quilaqueo, 2006).

En relación con las dimensiones que abarca el kimeltuwün en estudios realizados entre el año 1998 y 2003 por Quintriqueo (2005), se destacan cinco principios que guían la formación: 1) el kümeyawal ta che, definido como actitud y relación de respeto que idealmente se debe establecer entre los miembros de la familia y las comunidades mapuches; 2) el yamüwal ta che, representado por una relación de estima y de reconocimiento entre personas de un mismo grupo parental y de la comunidad mapuche en general; 3) el küme rakizuam ta nieal, como principio que considera el conocimiento de formación que permite asumir la concepción de persona al interior de la familia y en la comunidad; 4) el küme che geal, que se reconoce como enfoque evaluativo para determinar si la persona actúa solidariamente dentro del grupo parental; 5) y el kim che geal, principio que guía la formación de la persona sustentada en el conocimiento.

El principio kim che geal recurre a la noción ajkütual ta zugu (escuchar para comprender), como medio para evaluar cuando un niño o niña es capaz de estar atento y comprender lo que se le enseña. Del mismo modo, se utiliza el concepto kümeke gübam ta yenieal para referirse a la persona adulta capaz de entregar una buena formación valórica mediante un consejo o gübam. Este conjunto de contenidos y principios educativos para la formación de niños y niñas, constituye los saberes y conocimientos propios que aún son posibles de reconocer en la educación familiar mapuche. También se consideran en este trabajo algunos estudios exploratorios que definen categorías de contenidos del conocimiento educativo mapuche (Quilaqueo, 2003, Quilaqueo y San Martín, 2008; Quintriqueo y McGinity, 2008). Asimismo, se dispone de estudios de estrategias didácticas y peda-

\footnotetext{
${ }^{4}$ El yimümün se define como un proceso intencionado para instruir y formar a los niños y las niñas, para entregar una educación fundamentada en el patrimonio cultural mapuche. El yewmewün se entiende como una preparación de las personas para desenvolverse en un oficio, ejercitado para desenvolverse con agilidad. El mümülkan se concibe como una actividad educativa que tiene por objeto dar forma (estructurar) a un conjunto de contenidos para la enseñanza-aprendizaje (Quilaqueo y Quintriqueo, 2007)
} 
gógicas desde un enfoque intercultural susceptibles de utilizar en contextos indígenas (Valiente, 1993; Abdallah-Pretceille, 1996; Quilaqueo, Quintriqueo y Cárdenas, 2005).

Con respecto a la educación chilena, se constata que ésta pasa por un proceso de innovación y adaptación. Todo ello supone una aceptación de transformaciones estructurales internas y una negociación permanente de los equilibrios sociales, donde la escuela constituye un lugar privilegiado en los procesos de socialización para el aprendizaje de valores, de las normas y de los roles sociales (Perrenoud, 1998, 2002). Además de la escuela, están las otras instancias de socialización como el medio familiar, profesional y la misma sociedad del conocimiento (Forquin, 1997). En estos contextos, los principales obstáculos que encuentran los niños y niñas indígenas son los estereotipos y los prejuicios en el desarrollo de sus estudios (Rojas y Sepúlveda, 2002; Quilaqueo y Merino, 2003; Quilaqueo, Merino y Saiz, 2007; Saiz, Merino y Quilaqueo, 2009). Asimismo, se observan dificultades históricas de relaciones con el Otro, propias de una sociedad cada vez más segmentada socialmente (Cantoni, 1978; Todorov, 1993; Wieviorka, 1996; Schnapper, 1998). No obstante, se observa también que un currículum intercultural podría organizar y sistematizar contenidos educativos propios de los educandos a partir de la socialización del medio familiar y comunitario, como vía alternativa de investigación propuesta por Molina (2002), desde una sociología de la educación intercultural. Esto se fundamenta en el hecho de que la socialización del niño o niña mapuche, después de la familia, necesita junto con los no mapuches una escolarización más contextualizada para establecer relaciones interétnicas e interculturales más equilibradas (Loslier, 1997; Quilaqueo, 2007).

\section{Metodología}

El diseño utilizado para esta investigación es cualitativo. Este enfoque permite un intento de comprensión global y conceptualización del estudio del conocimiento educativo vernáculo mapuche. De acuerdo con Ruiz (1996), se tienen en cuenta dos características de este tipo de diseño. La primera obliga a tener una visión global del fenómeno que se va a estudiar, donde cada objeto de investigación puede ser entendido como un texto en un contexto, debiendo ser abordados ambos en su totalidad. La segunda característica es la que impulsa a no perder contacto con la realidad inmediata, puesto que la proximidad es un requisito indispensable. Para el desarrollo de esta investigación, se parte del núcleo temático de los saberes educativos mapuches por parte de una muestra de kimches orientada a descubrir, captar y comprender una teoría, una explicación, un significado que permita reconstruir el proceso de formación de personas y la representación de la educación mapuche. Este diseño se apoya en la Teoría Fundamentada (Grounded Theory) de Glaser \& Strauss (1967), que es definida por Strauss y Corbin en 2002 como “...una teoría derivada de los datos recopilados de manera sistemática y analizados por medio de un proceso 
de investigación” (2002:13). Señalando al respecto que: “...una teoría denota un conjunto de categorías bien construidas...” (2002: 25); por ejemplo, en temas y conceptos interrelacionados de manera sistemática por medio frases que revelan relaciones, con la finalidad de construir un marco teórico que explica algún fenómeno social, psicológico y educativo.

Para el procedimiento de interpretación de los datos se pueden distinguir tres tipos de codificación: abierta, axial y selectiva. En este trabajo, la interpretación se inicia con la codificación abierta, entendida como proceso en el que el investigador aborda el texto, con el fin de descubrir los conceptos, ideas y sentidos que él contiene. Trinidad, Carrero y Soriano (2006: 47), señalan que esto se produce “...cuando el investigador comienza a fragmentar los datos, en tantos modos como sea posible, con el objetivo de generar un conjunto de categorías...”, como categorías relevantes en la integración de una teoría. Para esta investigación, mediante la codificación abierta, los datos han sido disgregados, examinados y comparados en términos de sus similitudes y diferencias. Este trabajo es profundamente inductivo, por lo que no precisa de una teoría para aplicar sus conceptos, leyes o dimensiones en el texto que se está codificando. Por el contrario, el investigador debe "sumergirse" en el texto para identificar los conceptos, dimensiones y construir categorías y subcategorías pertinentes a la investigación (Strauss y Corbin 2002).

En síntesis, con el fin de comprender los contenidos de los métodos educativos mapuches, es necesario elaborar un cuerpo conceptual que se articule a través de proposiciones lógicas que lleven a la construcción de una teoría, ya que teorizar no sólo involucra desarrollar o descubrir ideas (conceptos), sino que además formularlas mediante un modelo lógico, sistemático y explicativo. Así, una teoría implica un conjunto de categorías bien construidas, es decir, la interrelación de temas y conceptos a través de proposiciones que indican relaciones con la finalidad de formar un marco teórico (Strauss y Corbin 2002). Sin embargo, de acuerdo con lo anterior, se debe aclarar que este marco teórico proviene de un modelo descriptivo propio y tradicional del enfoque, de tipo investigativo cualitativo, a un modelo conceptual teórico pertinente a los objetivos del estudio, lo que implica mayor capacidad de abstracción e integración de los resultados y hallazgos a otros contextos educativos similares. En este sentido, la conceptualización resultante del trabajo con la Teoría Fundamentada va más allá de un método descriptivo y sus limitaciones asociadas a la fiabilidad y generalización (Trinidad, Carrero y Soriano, 2006).

Para la recopilación de los datos, se aplicó una entrevista semiestructurada. La primera parte de la entrevista se centró en la percepción global del entrevistado sobre las características de la educación mapuche. La segunda parte, develó las experiencias personales de los kimches como formadores de los saberes educativos mapuches. Las entrevistas fueron aplicadas en mapunzugun por los investigadores. Los parti- 
cipantes fueron contactados mediante organizaciones mapuches y a través de familiares de estudiantes de la carrera de Pedagogía Intercultural en Contexto Mapuche de la Universidad Católica de Temuco.

El corpus está compuesto por los discursos de 30 kimches de las territorialidades Lafkenche, Naqche, Pehuenche y Wenteche de la región de la Araucanía, a quienes se les aplicó una entrevista semiestructurada. Los kimches entrevistados pertenecen a las comunidades de Huapi, Traiguén, Trapilhue, Paliwepillán, Sahuelhue, Cumcumñaqui y Huentelar. Las entrevistas fueron transliteradas al castellano formal. Las razones teóricas para trabajar con esta muestra son tres. Primero, el conocimiento educativo está validado en la memoria colectiva mapuche que los kimches conocen. Segundo, los conocimientos de los kimches permiten dar una interpretación adecuada sobre el saber educativo presente en las familias mapuches de dichas territorialidades. Tercero, al describir los principales saberes educativos se apela a los valores desde la lógica cultural mapuche que subyace en el discurso de los kimches (Quintriqueo, 2002).

El análisis de la muestra tiene en cuenta la lógica de construcción de conocimiento que se manifiesta en el discurso en mapunzugun de los kimches (Quilaqueo, et al., 2004), donde los contenidos educativos se ordenan en categorías procedimentales, actitudinales y conceptuales (Coll, 1994; Bixio, 1997). Además, como estudio de problemas sociales relevantes, desde la perspectiva del análisis del discurso de Wodak (2003) éste considera una postura crítica e interdisciplinaria, ya que se tiene en cuenta particularmente la relación existente entre el discurso y las comunidades en un contexto socioeducativo. Al mismo tiempo, puede fijarse en todos los niveles y dimensiones del discurso. Por último, descubre lo implícito (Van Dijk, 1984), lo que está escondido que por algún motivo no es inmediatamente obvio en las relaciones de dominación discursiva o de sus ideologías subyacentes. En la aplicación de las entrevistas a los kimches el argumento metodológico se fundamenta en la saturación empírica de contenido (Valle, 1997; Pires, 1997). De acuerdo con la metodología cualitativa, las categorías conceptuales del conocimiento educativo representan y reenvían a las otras dimensiones presentes en el discurso de los kimches, es decir, reenvía a su localización en un continuum de propiedades valóricas presentes en la memoria (Halbawch, 1970; Sabourin, 1997).

\section{Algunos resultados}

Las categorías reveladas con mayor frecuencia son: Prácticas Socioculturales, Respeto, Mapunzugun, y Kimeltuwün (Ver Tabla $\mathrm{N}^{\circ} 1$ ). Estas categorías se refieren a contenidos educativos que, mediante un diálogo cultural mapuche-no mapuche, podrían ayudar a contextualizar la escolarización de los alumnos y alumnas de escuelas situadas en las comunidades y los nuevos contextos de vida mapuche. 
Tabla 1: frecuencia de uso de las categorías prácticas socioculturales, respeto, mapunzugun y kimeltuwün.

\begin{tabular}{|lr|lr|ll|lr|}
\hline \multicolumn{2}{|c|}{$\begin{array}{c}\text { Prácticas } \\
\text { socioculturales }\end{array}$} & \multicolumn{2}{c|}{ Respeto } & \multicolumn{2}{c|}{ Mapunzugun } & \multicolumn{2}{c|}{ Kimeltuwün } \\
\hline Günechen & $28,6 \%$ & Günechen & $41,1 \%$ & Saber escuchar & $28,4 \%$ & Gübam & $25,6 \%$ \\
\hline Gijatun & $28,2 \%$ & Personas & $27,6 \%$ & Transmisión oral & $22, \%$ & Enseñanza familiar & $22,5 \%$ \\
\hline Pentukuwün & $13,3 \%$ & Familia & $11,1 \%$ & Valorarla lengua & $22,1 \%$ & Actitud aprendizaje & $15,0 \%$ \\
\hline Tuwün & $11,4 \%$ & Naturaleza & $9,4 \%$ & Enseñanzakimche & $11,0 \%$ & Enseñanza kimche & $15,0 \%$ \\
\hline Küpan & $3,3 \%$ & Ceremonias & $5,8 \%$ & Saber comportarse & $6,5 \%$ & Actitudenseñanza & $10,6 \%$ \\
\hline Wewpin & $7,2 \%$ & Feyentunzugu & $3,0 \%$ & Expresión oral & $5,0 \%$ & Enseñanza mapunzugun $7,9 \%$ \\
\hline Mafün & $8,1 \%$ & Escuchar & $2,0 \%$ & Reflexión & $5,0 \%$ & Ser mapuche & $3,4 \%$ \\
\hline
\end{tabular}

Fuente: datos obtenidos mediante de la codificación con el software Atlas-ti 5

\section{Prácticas socioculturales}

Los kimches señalan que el conocimiento se construye por medio de prácticas sociales y culturales. Para ejemplificar esta categoría, se considera la ceremonia socio religiosa gijatun (rogativa) con una frecuencia de uso de $28,2 \%$ y el saludo tradicional pentukuwün (saludo protocolar) con una frecuencia de 13,3 \%. El gijatun y el pentukuwün son prácticas socioculturales que se refieren a dos formas de socialización de los saberes educativos. En el gijatun, la formación se desarrolla de manera individual y grupal. Al respecto, los kimches naqches (del valle) y kimches pewenches (de la Cordillera de los Andes), reconocen un tipo de gijatun individual y otro colectivo. El gijatun individual, que puede incluir a la familia en algunos casos, es una ceremonia que se desarrolla diariamente para invocar a günechen (considerado como espíritu superior por los mapuches) por sucesos emergentes y de la vida cotidiana. A este gijatun los kimches pewenches lo denominan püntefun. Se practica frente al fogón o frente de la casa, al lado oriente y se les muestra a los niños y niñas el contenido, la forma y el rigor de su realización. Por su parte el gijatun colectivo tiene características de xawün o reunión y su organización implica la participación de varias familias. Este tipo de gijatun se denomina kamarrikun en las comunidades naqches y se prepara generalmente durante un año.

Entre los testimonios, se hace hincapié en que el gijatun tiene como objeto “...generar la interacción entre el medio natural, social y cultural con günechen, donde se busca que él le entregue a los seres humanos los elementos necesarios para desarrollarse...” (E3; 2) ${ }^{5}$. Los kimches de la zona lafkenche explican que las normas socioculturales guían la formación de las personas, como es el caso del gijatun, ya que por medio ellas los niños y niñas logran actitudes para construir y mantener el saber y conocimiento de la comunidad. Se manifiesta la actitud de respeto en las ceremonias, allí “....todo los participantes deben seguir un orden de acuerdo con el

${ }^{5}$ (E3; 2): E3 corresponde a la entrevista número 3 y el número 2, a la página donde se encuentra la información. 
contenido y la actitud de las personas para agradecer o invocar a günechen" (E3; 6). Cada ceremonia se desarrolla desde la perspectiva de un saber ancestral, esto implica enseñar el procedimiento y el contenido a los niños y las niñas. De esta forma, se regula la participación de las personas durante su desarrollo y se concluye que la enseñanza de las prácticas socioculturales es principalmente de tipo actitudinal. En efecto, se constata que la referencia a günechen es la de mayor frecuencia con un 28,6 \%. Sin embargo, si consideramos que la ceremonia gijatun se desarrolla en torno a la concepción de günechen, en conjunto, ambas categorías alcanzan una frecuencia de uso del 56,8 \% como contenido educativo. Estos aspectos se manifiestan mediante su referencia explícita o implícita en los discursos de los kimches. Lo que se destaca en los conocimientos que se entregan a los niños y niñas es el rigor para llevar a cabo la interacción entre el medio natural, social y espiritual, es decir, los contenidos actitudinales de la ceremonia como saber respetar y saber escuchar, pero recurriendo a normas y reglas establecidas ancestralmente.

Con respecto al pentukuwün, se trata de un saludo protocolar que se describe como una práctica de socialización del conocimiento sobre el küpan (ascendencia paterna) y el tuwün (ascendencia materna y territorial) de cada persona, pero, que al mismo tiempo, se reconoce como perteneciente a una misma comunidad sanguínea. El küpan se define como un conocimiento de la relación de parentesco de la ascendencia paterna y de un oficio. Se fundamenta en la memoria histórica de cada familia y su comunidad. Por su parte, el tuwün se reconoce como la procedencia geográfica materna del origen de las personas. Estos conceptos se reconocen en una práctica discursiva que tiene por objeto saber quien es el interlocutor, por ejemplo, cuando se expresa que “...el küpan tiene relación con la familia, en mi caso mi mamá era Llaulén y mi papá es Rapi...” (E23; 7). Aquí, el küpan de cada persona tiene directa relación con los apellidos, mientras que en el tuwün, se ejemplifica señalándose que “...mi papá tiene su tuwün en Kajaki, él es Kajaki che [gente del lugar Kajaki]...”(E23; 7).

Los contenidos educativos tuwün y küpan, alcanzan una frecuencia de 11,4 y 3,3 \% respectivamente, es decir, el contenido educativo del concepto pentukuwün alcanza el 13,3\%, lo cual se relaciona con los aspectos actitudinales y procedimentales. Estos contenidos son los que se transmiten, desde la educación mapuche, a los niños y niñas para enseñarles su origen parental y el lugar de procedencia. En síntesis, en el saludo protocolar pentukuwün el contenido enseñado es más de tipo conceptual.

\section{Respeto (a günechen, las personas y la naturaleza)}

El respeto, como contenido formativo para ser persona (che en mapunzugun), se presenta subordinado a las subcategorías günechen, la persona y la naturaleza; representando el 78,1 \% de frecuencia para referirse a la educación mapuche. Se manifiesta, por ejemplo, entre los kimches de comunidades naqches que “...en el respeto hacia las personas mayores 
se considera el saber que poseen niños, niñas y jóvenes como resultado de su formación...” (E6; 1). Se subraya que, a partir de la idea del kuyfike zugu o saber ancestral, el respeto hacia las personas adultas mayores se mantiene, porque “.... son las que poseen y transmiten los principales saberes ancestrales. Son kimches por su mayor conocimiento y relación espiritual con günechen" (E2; 11). En consecuencia para definir el respeto se utiliza el concepto yamüwün (respeto). Este concepto se identifica principalmente como contenido actitudinal, porque se señala que “...la valoración de la edad, el respeto por su función y posición social que ocupa la persona en la comunidad...” (E4; 8), es la que determina el tipo de relación que se debe tener.

Los kimches enfatizan que todo el conocimiento que poseen las personas se debe al saber que ancestralmente han heredado, fundamentalmente, la relación espiritual con günechen. Esto se manifiesta de manera transversal en el discurso educativo y se confirma como el aspecto que se presenta con mayor frecuencia y que alcanza el $41,1 \%$. Sin embargo, al mismo tiempo señalan que cada persona tiene la posibilidad de ser kimche. En lo anterior radica la necesidad de formar a niños, niñas y jóvenes para que adquieran el conocimiento cultural como condición para ser personas. Se pone de manifiesto que los kimches son los que han cultivado históricamente los conocimientos y saberes culturales, por ejemplo, lo que dice relación con el territorio de procedencia de la familia con diferente küpalme (linaje) y los valores sobre el respeto por el entorno natural, social y espiritual. De esta forma, los niños, niñas y jóvenes que se dirigen a los kimches deben demostrar el respeto a la persona por su ascendencia. En tanto, el saber actitudinal, que se construye socialmente, se presenta con una frecuencia de 27,6 $\%$, principalmente para referirse a los adultos. Asimismo, el respeto se asocia "...con las personas mayores que poseen el conocimiento del tronco familiar y el proceso de asentamiento en territorios ancestrales" $(\mathrm{E} 12,1)$. Los kimches se refieren a los niños, niñas y jóvenes como el centro de su preocupación, pero siempre en interacción con su medio social, natural y una marcada espiritualidad (en relación con günechen), ya que permite a las personas estar en equilibrio y en unión con los aspectos socioculturales ancestrales.

En relación con el respeto hacia las personas “adultas mayores”, éste se asocia también el concepto escuchar o ajkütuwün. El ajkütuwün se define como saber escuchar, concepto utilizado con una frecuencia de $2 \%$ para la categoría respeto. Se trata de un saber actitudinal y procedimental que facilita el desarrollo de la cualidad del niño o niña en relación con el saber y el conocimiento, lo que permite una comprensión de lo que se enseña. Es un proceso complejo que requiere concentración, donde el receptor debe tener una buena disposición para interpretar y comprender el significado del mensaje. En efecto, en la lógica mapuche del saber escuchar incluye los siguientes aspectos con el objeto de desarrollar y comprender las relaciones afectivas del proceso formativo: disposición cognitiva, concentración, determinada pos- 
tura corporal, selección de la información y concreción de lo aprendido mediante acciones. Todo esto implica tener cierta disposición sobre determinadas realidades socioculturales y comprender códigos sociales y culturales, por ejemplo: “...al hijo mayor de la familia se le da la oportunidad de escuchar y se le lleva donde van los mayores. Se le dice: tú tienes que ir a escuchar esta conversación... Si yo algún día me muero, tú vas a llevar estos conocimientos...” (E16; 8). De esta manera, saber escuchar los consejos que transmiten las personas mayores le permite al niño, a la niña y al joven aprender y comprender cómo formarse y transformarse en che o persona. Lo que se desprende de lo anterior es que de esta forma podrá compartir los principios valóricos con sus pares y mostrar lo aprendido a sus hermanos menores. Así, el niño o niña que sabe escuchar podrá reproducir lo enseñado por las personas mayores sobre saber ancestral, destacándose la obediencia y el respeto hacia los ancianos como un comportamiento que le permitirá actuar de acuerdo con las reglas y normas de su familia y de su comunidad.

En la categoría de contenidos sobre el medio natural subyace el aspecto valórico expresado en el concepto feyentun zugu con una frecuencia de un $3 \%$. El feyentun se define como el valor que le da una persona a las orientaciones entregadas por los füshakeche (anciano o anciana con características de sabio), ya que transmiten el legado cultural ancestral. Por esta razón se manifiesta que “...se considera importante que los niños y las niñas desarrollen el respeto y lo lleven a la práctica demostrando obediencia a los füshakeche...” (E12; 3). Sin embargo, en las ideas de los kimches se expresa también que “...los niños, niñas y jóvenes están olvidando este valor...” (E4; 2). Se señala que “...prefieren otras enseñanzas y que guían su vida desde pensamientos generalmente superficiales, carentes de sentido cultural y social para el mapuche..." (E4; 2).

Desde una perspectiva socio religiosa, el feyentun adquiere una connotación espiritual, dado que los mapuches admiten la existencia de un espíritu superior. El espíritu superior günechen sería el que ejerce una influencia en el comportamiento de cada persona. Esto significa que para el mapuche, lo espiritual es vital para su desarrollo social y cultural como persona y como comunidad. La connotación espiritual se plasma en ceremonias socio religiosas como el gijatun o jejipun. De esta manera, el feyentun para los kimches es una actitud que se debe desarrollar en niños, niñas y jóvenes. Este es un contenido de tipo actitudinal y procedimental que entregan los padres con la evaluación de los miembros de su comunidad, particularmente de un kimche, por ejemplo: “...cuando se casa un hijo debe ser guiado por su mamá y su papá... No está separado de los padres, viven juntos o muy cercanos a ellos. Los abuelos se hacen cargo de formar a sus nietos..." (E26; 1). La formación que entregan los abuelos permite el aprendizaje de las normas de conducta del medio familiar y comunitario. En este sentido, la familia extendida permite la formación de las nuevas generaciones por parte de los abuelos, porque guían a sus nietos de acuerdo con sus propios saberes y los saberes adquiridos en las relaciones interétnicas de la comunidad con la 
sociedad no mapuche. Los saberes que los abuelos han enseñado históricamente a sus nietos se han entregado mediante relatos sobre la memoria familiar de cada comunidad; sin embargo, los nuevos contextos de vida han deteriorado el proceso de formación actitudinal.

\section{Mapunzugun en la educación mapuche}

Los kimches señalan que el mapunzugun es la lengua propia y que constituye una herramienta fundamental para enseñar los saberes mapuches. Esta categoría está formada por los códigos “saber escuchar”, “transmisión oral”, “valor de la lengua”, “enseñanza kimche”, “saber comportarse”, “expresión oral”. Los códigos que se presentan con mayor frecuencia son: saber escuchar con un 28,4\% y su transmisión con el 22,1\%. Esto significa que la concepción de mapunzugun que utilizan los kimches está relacionada con la interacción que se establece entre un niño, una niña o un joven con un adulto, puesto que la oralidad ha sido el único medio que han utilizado para su enseñanza. Esta oralidad alcanza un $27 \%$ de frecuencia si se suma con el código expresión oral con 5\%. El nivel de frecuencia es muy cercano al del concepto saber escuchar, con 28,4\%. Así, se le considera un saber para la formación de persona “...ya que los niños y niñas se crían bien al formarlos con su lengua mapunzugun...” (E23; 4). Esto se relaciona con el concepto valorar la lengua que alcanza una frecuencia de uso de 22,1 \% en el discurso de los kimches. De esta forma, en el discurso de los kimches, el mapunzugun es un elemento fundamental para la socialización de los saberes educativos, debido a que es un saber que permite enseñar contenidos socioculturales en la formación de niños y niñas, en la relación espiritual con günechen y en la relación entre los miembros de una comunidad.

El valor del mapunzugun para la formación de niños, niñas y jóvenes, en general, en las territorialidades que se consideran en este trabajo, se plantea como conocimiento necesario para interactuar con el medio social y cultural a través de la tradición oral (Bernal, 2000). Esto implica recurrir a un lenguaje ontológico construido en la relación de la persona mapuche con el medio natural, social y cultural en el cual se encuentra inserto (Fernández, 1999; Ong, 2006). En este sentido, la lengua mapunzugun es un saber que permite la reproducción de la cultura. En efecto, el mapunzugun se define como un componente cultural de socialización transversal. De este modo, tanto el güxam (conversación para interactuar en relación a un contenido) como el gübam (método para transmitir lecciones actitudinales para lograr adquirir un saber educativo) son herramientas de socialización para la formación de niños y niñas, pero particularmente de los jóvenes. Este proceso permite transmitir significados y representaciones lógicas del mundo desde la propia perspectiva sociocultural por medio de prácticas socioculturales tales como el ajkütuwün. El mapunzugun como elemento transversal de la educación mapuche abarca las categorías de contenidos conceptual, procedimental $\mathrm{y}$ actitudinal. 


\section{Kimeltuwün (acción educativa)}

La categoría kimeltuwün (de acuerdo con la Tabla 1) está compuesta por siete códigos o conceptos que la definen: "gübam”, "enseñanza intrafamiliar”, "actitud de aprendizaje”, "enseñanza kimche”, "actitud de enseñanza”, “enseñanza mapunzugun” y "ser mapuche”. Los que se presentan con mayor frecuencia son el gübam y enseñanza intrafamiliar con $25,6 \%$ y $22,5 \%$ respectivamente. El gübam, en el discurso de los kimches de comunidades wenteches, es la forma por la cual se transmiten y fortalecen los saberes, es decir, es un método que considera un discurso actitudinal especializado que se desarrolla mediante un contenido sociocultural, ya que “...se dirige al comportamiento de las personas y permite el desarrollo del conocimiento mapuche en el tiempo" (E5; 10). Desde este punto de vista, sólo se considera formada (bien educada) a la persona que actúa privadamente y públicamente demostrando los saberes y conocimientos adquiridos en un gübam. Los contenidos del gübam se orientan al desarrollo de la obediencia, saber escuchar, el respeto que los niños las niñas y los jóvenes deben tener hacia las personas adultas de la comunidad, los miembros del tronco parental y los miembros de la sociedad en general.

Con respecto a la importancia de la adquisición de nuevos conocimientos, por medio del kimeltuwün, se describe la sabiduría de los kimches, indicando que “...tienen sus conocimientos en la cabeza igual que en los libros. Todo el tiempo han hecho weupin para desarrollar el conocimiento... los kimches confrontan el conocimiento con otro kimche para aprender y validar lo que piensan...” (E10; 1). Sin embargo, subyace en el discurso de los kimches, en el contexto actual de las familias y de las comunidades, la necesidad de adquirir nuevos conocimientos de la sociedad occidental para comprender los nuevos contextos de la vida mapuche.

Entre los kimches de la zona Lafkenche, Naqche y Wenteche, el kimeltuwün es comprendido como un proceso de "aprendizaje y enseñanza”6 de contenidos sociales y culturales. El énfasis en los contenidos culturales mapuches se presenta como el principal argumento para ser che o ser persona. Esto quiere decir que la formación se orienta para que los niños, niñas y jóvenes interactúen en su familia, en su comunidad y en la sociedad chilena. Este es un enfoque educativo que se está organizado con el objetivo de entregar una formación sobre la base de contenidos de aprendizajes reglamentados por el $\boldsymbol{a z}$ o costumbre consuetudinaria mapuche (Housse, 1938).

En las entrevistas, se observa que los conceptos 'actitud de aprendizaje' y 'enseñanza kimche' alcanzan la frecuencia de $15 \%$ cada uno. Se pone énfasis en que el aprendizaje-enseñanza se planifica como acción educativa con el objeto de transmitir saberes y conocimientos. De esta manera, el kimeltuwün constituye un principio para enseñar contenidos educativos en la formación de

\footnotetext{
${ }^{6}$ Entre los kimches se pone énfasis en el concepto de aprendizaje, porque sólo puede enseñar el que puede aprender (Quilaqueo, 2006).
} 
niños, niñas y jóvenes. Se resalta que el kimeltuwün tiene una finalidad relacionada con las acciones educativas que realizarán las futuras generaciones, para el desarrollo del saber y del conocimiento mapuche. Se manifiesta que “...siempre, en una comunidad, hay una persona sabia que tiene mucho conocimiento y un buen pensamiento... Se le reconoce y se toma como ejemplo, puesto que ha sido formado como mapuche” (E10; 8). Esta intervención se relaciona directamente con, la calidad de kimche.

En síntesis, la acción educativa kimeltuwün se lleva a cabo de acuerdo con el desarrollo intelectual y físico de cada niño, niña o joven, ya que “...a medida que va creciendo se le incorpora a actividades que desarrollan las personas adultas...” para que de esta manera se pueda evaluar su desarrollo y se concluye con la afirmación “... después se dirá tiene buen pensamiento o tiene mal pensamiento” (E10; 2). De la intervención anterior, se puede observar que la evaluación expresada por los kimches es actitudinal, ya que se expresa que “...una vez adulto podrán actuar de acuerdo con las normas de las comunidades y las normas de la sociedad occidental aprendida en la escuela" (E10; 2). En esta última afirmación, los kimches aluden a la escuela preocupados por la educación que se entrega frente a los nuevos contextos sociales y culturales.

\section{Discusión}

Los kimches se refieren a los saberes educativos como un discurso directo que se fundamenta en una argumentación relacionada con el tiempo ancestral (kuyfi). Se trata de un relato argumentativo cuyo punto de inicio lo constituye el tema (zugu en mapunzugun), sobre el cual se razona apoyado por fundamentos y evidencias desde un contenido. El contenido se desarrolla basado en uno o varios hechos, cada uno se presenta como un problema y la forma en la que éste se resuelve. La presentación de los hechos culmina con una conclusión o moraleja, de la cual los niños, niñas y jóvenes en formación pueden hacer una evaluación y extraer una lección (Housse, 1938). Cada hecho tiene lugar en un contexto determinado, esto es una situación, lugar y circunstancias que se localizan ya sea en el tiempo actual o en el pasado, mediante la memoria social ancestral o kuyfike zugu. Se trata de referencias a los consejos, valores, hábitos y costumbres; cuyo rol es desarrollar la cultura, la lengua mapuche y enseñar a los más jóvenes a relacionarse sin renunciar a su identidad.

Para los kimches, el aprendizaje de los niños, niñas y jóvenes, que se lleva a cabo en una interacción con el medio social y natural, es un proceso que se realiza en el medio familiar semejante a la función que cumple la escuela. Cuando se refieren a la escuela, en sus discursos subyace la socialización y el tipo de escolarización que ellos mismos recibieron. La escolarización es entendida como la ocasión para dotar de conocimientos acerca de los valores y las normas necesarios para la integración a la sociedad chilena. Sin embargo, dicha escolarización no considera, los conocimientos de la socialización familiar, puesto que se observa un trato discriminatorio 
explícito e implícito al ser tratados como indios (Quilaqueo, Merino y Saiz, 2007). Y, desde la crítica epistemológica planteada por Van Haecht (1999), se presenta un problema de paradigmas para analizar el conocimiento de los saberes educativos mapuches. En efecto, el docente que trabaja con alumnos de origen indígena, generalmente, no está formado en conocimientos culturales y sociales de sus comunidades. Esto implica que aunque haya un avance en la escolarización y un cambio en la relación con los indígenas, la escolarización sigue teniendo "... una serie de limitaciones que no ha podido superar al no prescindir, en último término, de la ideología monocultural y, de alguna manera, etnocéntrica” (Molina, 2002). La escuela chilena ha sido monocultural y se ha utilizado como método de colonización y de formación del Estado. Esto ha provocado una disminución o exclusión del uso de los saberes educativos mapuches en el medio familiar, provocando en muchos casos la autonegación de la identidad mapuche por parte de los jóvenes para poder integrarse a la sociedad chilena (Quintriqueo y Maheux, 2004).

Se señala que la educación mapuche crea espacios de interacción entre las personas como medio de socialización, entendida como acción que facilita la comprensión de las normas y creencias establecidas por la sociedad (Rocher, 1992; Vásquez y Martínez, 1996). Esta interacción se expresa como confrontación de saberes (en algunos casos se asocia al concepto de weupin), cuya finalidad es la comprensión, el aprendizaje y su evaluación. La confrontación de saberes es expresada como el intercambio y construcción de conocimiento de manera reiterativa con sustento en la memoria social kuyfike zugu. En este proceso, se induce a los niños, niñas y jóvenes a utilizar el principio de "aprender a escuchar" o ajkütual ta zugu, como medio de formación. Sin embargo, se puede constatar también que la confrontación señalada entre las personas adultas y jóvenes incluye saberes y conocimientos de tipo occidental y mapuche para comparar su contenido y el valor que representa para ellos.

En general, se puede constatar que cuando se hace referencia a la interacción con el medio sociocultural y natural, se pone énfasis en los valores. De esta manera, el valor de la naturaleza (medio ambiente natural) supone un estrecho vínculo entre la persona y el territorio en el cual habita, que se expresa como respeto. En mapunzugun este respeto por la naturaleza se denomina $\boldsymbol{e}$ kuwün, más precisamente como el respeto tanto por las personas como en su relación con la naturaleza (Paillalef, 2003), ya que se cree que ésta tiene su propio newen (fuerza de vida) y un gen (un espíritu) que la cuida. Así, en relación con lo anterior, entre los mapuches, es común, por ejemplo, observar una actitud de respeto hacia un mawiza (monte con abundante vegetación herbácea y arbórea), un menoko (fuentes de agua con reservas de plantas medicinales), un lewfü (río), el lafken (mar) y un zegüñ (volcán), (Cavières, Macheo, Meza y Pañinao, 2005).

En síntesis, los saberes educativos mapuches, considerados vitales para el desarrollo de la educación mapuche, se encuentran en peligro. Esta situación se debe a que desde el punto de vista de un kimche y su comunidad, a partir de la experiencia de haberse integrado a la sociedad chilena se 
manifiesta que“...falta el eje central, se ha ido perdiendo lo que es nuestro conocimiento, la historia de la concepción de la vida mapuche, la cosmovisión de todo un conjunto de valores que hacen el ser de lo que nosotros somos los mapuche como pueblo" (E $17 ; 2)$. En efecto, en las observaciones realizadas, los kimches imputan a la escuela los problemas que enfrenta la educación mapuche. La castellanización y la monoculturalidad son las principales políticas de escolarización que obligaron a abandonar la lengua mapuche. Desde entonces, este sería el motivo principal de que la vitalidad del conocimiento cultural mapuche y su importancia en la formación de personas estén en riesgo actualmente.

\section{Conclusiones}

El análisis de los discursos de los kimches ha revelado contenidos educativos que muestran la existencia de categorías de saberes y conocimientos mapuches para la formación de niños, niñas y jóvenes. Los conocimientos que prevalecen, se refieren a la formación que incide en la relación entre los seres humanos, su entorno natural y espiritual. En este proceso, es necesario saber tratar de manera transversal los aspectos espirituales, fundamentalmente aquellos que dicen relación con el espíritu superior günechen; puesto que los kimches deducen que es él quien gobierna a las personas y su relación con el medio natural.

La formación mapuche considera saberes actitudinales que le permiten a la persona relacionarse con el medio social y natural sobre la base de normas y reglas construidas en la relación persona-günechen-medio natural de las comunidades. Se constata que esta es la relación esencial en todo proceso de construcción de saberes y conocimientos mapuches, porque permite tener una concepción de mundo que los distingue de otras comunidades. Esta concepción de mundo es creada y recreada mediante una lengua propia, y construida en la relación persona-günechen-medio natural, cuyo objetivo es dar sentido a los conocimientos propios y a los no mapuches. Desde el punto de vista del discurso de la memoria social de los kimches, éste se construye sobre la base de una tradición argumentativa basada en la oralidad, es decir, el uso de una modalidad discursiva construida sobre la base del güxam o conversación (tipo relato), que constituye el tipo de discurso más recurrente en la comunicación oral.

Finalmente, se revela que entre las familias y comunidades subsiste un tipo de socialización para la formación de niños, niñas y jóvenes, donde la persona que asume la posición de educador tradicional (kimche) es primordial. Además, se manifiesta por parte de los kimches que las dificultades que enfrentaron las distintas generaciones en el medio escolar, serían consecuencias del desconocimiento de los docentes de aula de los saberes socioculturales mapuches. En consecuencia, es urgente superar las dificultades de la escolarización descontextualizada a partir de una nueva relación con el saber, mediante una formación docente que considere la incorporación de contenidos culturales mapuches al currículum. 


\section{Bibliografía}

Abdallah-Pretceille, Martine (1996), Vers une pédagogie interculturelle. Anthropos, Paris.

Alchao, Yeny, Cariman, Alejandra, et al. (2005), "La formación de persona y el oficio en el saber y conocimiento educativo mapuche, desde la visión del kimeltuwün en el área territorial sur de la Novena Región”. Tesis de Licenciatura en Educación, Escuela de Pedagogía Básica, Universidad Católica de Temuco, Temuco.

Augusta, Felix (1903), Lecturas Araucanas. Editorial Kushe. Temuco, Chile.

Bengoa, José (1987), Historia del Pueblo Mapuche, siglos XIX y XX Ediciones Sur. Colección Estudios Históricos. Santiago de Chile.

Beillerot, Jacky (1998), "La relación con el saber: una noción en formación”, en Jacky Beillerot, Blanchard, Claudine y Mosconi, Nicole. Editorial Paidós SAICF, Buenos Aires.

Bejoit, Guy (2003), Todo cambia: Análisis sociológico del cambio social y cultural en las sociedades contemporáneas. LOM ediciones: Santiago de Chile.

Berger, Peter y Luckmann, Thomas (2001), La Construcción Social de la Realidad. Amorrortu Editores, Madrid.

Bernal, Guillermo (2000), Tradición oral, escuela y modernidad. La palabra encantada. Cooperativa Editorial Magisterio, Santafé de Bogotá.

Bixio, Cecilia (1997), Contenidos Procedimentales. Los procedimientos: su enseñanza, aprendizaje y evaluación. Homo Sapiens Ediciones, Rosario, Argentina.

Bonfil-Batalla Guillermo (1987), "La teoría del control cultural en el estudio de los procesos étnicos”, en Revista Papeles de la Casa Chata. Año 2, N³, pp. 36-52, México.

Bradford, Margaret (1976), "El sistema de valores del mapuche”, en Estudios antropológicos sobre los mapuches de Chile sur - central. (Recopilación e introducción: Dillehay, Tom). Pontificia Universidad Católica de Chile, Sede Regional Temuco.

Cantoni, Wilson (1978), "Relaciones del mapuche con la sociedad nacional chilena”, en Raza y Clase en la Sociedad Postcolonial. UNESCO. Organización de las Naciones Unidas para la Educación, la Ciencia y la Cultura, Madrid, pp. 227-334. 
Catrileo, María (1995), Diccionario Lingüístico-etnográfico de la lengua mapuche. Editorial Andrés Bello, Santiago de Chile.

Cavières, Ivonne, Macheo, Rafael, et al. (2005), "Valores educativos mapuches para la formación de persona, en el contexto familia-escuela-comunidad, desde la concepción que subyace en el discurso de los kimche y docentes de origen mapuche”. Tesis de Licenciatura en Educación, Escuela de Pedagogía Básica, Universidad Católica de Temuco, Temuco.

Coll, César (1994), “Los contenidos procedimentales”. En AAVV, Los contenidos de la reforma. Enseñanza y aprendizaje de conceptos, procedimientos y actitudes. Santillana, Buenos Aires.

Erize, Esteban (1960), Diccionario Comentado Mapuche-Español: Araucano, Pehuenche, Pampa, Picunche, Ranculche y Huilliche. Cuadernos del Sur. Instituto de Humanidades, Universidad Nacional del Sur. Buenos Aires.

Fairclough, Norman (1995), Critical Discourse Analysis: the Critical Study of Language. Longman, London.

Fernández, César (1999), Cuentan los mapuches. Ediciones Nuevo Siglo S. A., Buenos Aires.

Forquin, Jean Claude (1997), Les sociologues de l'éducation américains et britanniques. Présentation et choix de textes. De Boeck Université, Bruxelles.

Foucault, Michel (1997, $18^{\circ}$ edición), La arqueología del saber. Siglo XXI editores, México DF.

Glaser, Barney \& Strauss, Anselm (1967), The discovery of grounded theory. Aldine, Chicago.

Halbwachs, Maurice (1970), Morphologie sociale. Armand Colin, Paris.

Housse, Emile (1938), Une épopée indienne. Les Araucans du Chili. Librairie Plon, Paris.

Le Goff, Jacques (1991), El orden de la memoria, el tiempo como imaginario. Editorial Paidós, España.

Loslier, Sylvie (1997), Des relations interculturelles. Editions Liber, Québec.

Lyotard, Jean- Francois (1994), La condición posmoderna. Ediciones Cátedra, Madrid. 
MINEDUC (Ministerio de Educación de Chile), (2005), Orientaciones para la contextualización de Planes y Programas para la Educación Intercultural Bilingüe. Santiago de Chile.

Molina, Fidel (2002), Sociología de la educación intercultural. Vías alternativas de investigación y debate. Grupo Editorial Lumen Hvmanitas, Buenos Aires.

Montesperelli, Paolo (2004), Sociología de la memoria. Ediciones Nueva Visión, Buenos Aires.

Morin, Edgar (1998), Introducción al pensamiento complejo. Gedisa, Barcelona.

Ogien, Albert (2008), Las formas sociales del pensamiento. La sociología después de Witgenstein. Nueva Visión, Buenos Aires.

Ong, Walter (2006), Oralidad y escritura: Tecnologías de la palabra. Fondo de Cultura Económica, Tercera reimpresión, Buenos Aires.

Paillalef, Julio (2003), Los mapuches y el proceso que los convirtió en indios. Psicología de la discriminación. Universidad Tecnológica Metropolitana, Santiago.

Perrenoud, Philippe (1998), La construction de compétences à l'école. ESF, Paris.

Idem (2002), "Les conceptions changeantes du curriculum prescrit: hypothèses”. Educateur, Numéro spécial: Un siècle d’éducation en Suisse romande, $\mathrm{N}^{\circ} 1$, pp. 48-52.

Pires, Alvaro (1997), “Échantillonnage et recherche qualitative: essais théorique et méthodologiques", en Poupart, Jean et al. La recherche qualitative. Enjeux épistémologiques et méthodologiques, Gaëtan morin éditeur, Montréal, pp 256-297.

Quilaqueo, Daniel (1994), "Participation Traditionnelle et Participation Provoquée. Les nouvelles communautés mapuche de deux provinces d'Argentine: Neuquén et Río Negro”, en Mondes en Développement, de I'Institut de Mathématiques Appliquées ISMEA, tome 22, 1994, N86, Paris. Pp. $17-26$

Idem (2005), “Educación Intercultural desde la Teoría del Control Cultural en Contexto de Diversidad Sociocultural Mapuche”, en Cuadernos Interculturales, Año 3, $\mathrm{N}^{\circ}$ 4, enero-junio 2005. Centro de Estudios Interculturales y del Patrimonio (CEIP). Universidad de Valparaíso, pp. 37-50.

Idem (2006), "Valores educativos mapuches para la formación de persona 
desde el discurso de kimche”, en Estudios Pedagógicos XXXII, N²; Valdivia, pp. 73-86.

Idem (2007), "Saberes y conocimientos indígenas en la formación de profesores de educación intercultural”, en Educar em Revista, Curitiba, pp.223239.

Quilaqueo, Daniel y San Martín, Daniel (2008), “Categorización de saberes educativos mapuche mediante la Teoría Fundamentada”, en Estudios Pedagógicos XXXIV, N²; Valdivia, pp. 151-168.

Quilaqueo, Daniel y Merino, María (2003), “Estereotipos y prejuicio étnico hacia los mapuches en textos complementarios a la asignatura de Historia”, en Revista Campo Abierto, 2003, $N^{\circ} 23$, pp. 119-135. Universidad de Extremadura, España.

Quilaqueo, Daniel; Quintriqueo, Segundo; Catriquir, Desiderio y Llanquinao, Gabriel (2004), "Kimeltuwün mew amukey ta zugu. Una didáctica para abordar conocimientos mapuche en el proceso de formación inicial en educación intercultural”, en Grupo de Investigación, Cuatro estudios para mejorar la formación inicial docente. Facultad de Educación. Universidad Católica de Temuco, pp. 53-148.

Quilaqueo, Daniel; Quintriqueo, Segundo y Cárdenas, Prosperino (2005), Educación, currículum e interculturalidad. Elementos sobre formación de profesores en contexto mapuche. Facultad de Educación, Universidad Católica de Temuco. Frasis editores, Santiago de Chile.

Quilaqueo, Daniel; Merino, María y Quintriqueo, Segundo (2005), “Saberes mapuches y conocimientos educativos vernáculos transmitidos por kimches. Sistematización para una educación intercultural”. Proyecto Conicyt/ Fondecyt Regular, 2005-2007.

Quilaqueo, Daniel; Merino, María y Saiz, José (2007), “Representación social mapuche e imaginario social no mapuche de la discriminación percibida”, en Atenea, segundo semestre, año 2007 - N 496, pp. 81-103.

Quilaqueo, Daniel; Quintriqueo, Segundo (2008), “Formación docente en educación intercultural para contexto mapuche en Chile”, en Cuadernos Interculturales, primer semestre, año/vol. 6, numero 10, Universidad de Valparaíso, Viña del Mar, pp. 91-110.

Quintriqueo, Segundo (2002), “Representación de la relación de parentesco que la familia mapuche posee y su transmisión a niños mapuche de edad escolar primaria en relación con los contenidos de Formación Inicial Docente”. Informe de Tesis de Maestría en Educación. Université du Québec en Abitibi-Témiscaminque, Québec.

Idem (2005), “Implicancias de la escolarización en la construcción de la 
identidad cultural de alumnos mapuche en el medio escolar de la IX Región”, en Quilaqueo, Daniel, Quintriqueo, Segundo y Cárdenas, Prosperino (2005). Educación, currículum e interculturalidad. Elementos sobre formación de profesores en contexto mapuche. Facultad de Educación, Universidad Católica de Temuco. Frasis editores, Santiago de Chile, pp. 191-248.

Quintriqueo Segundo y Maheux Gisèle (2004), “Exploración del conocimiento sobre la relación de parentesco como contenido educativo para un currículum escolar intercultural en comunidades mapuches”, en Revista de Psicología, Vol. XIII, N 1, 73-91.

Quintriqueo, Segundo y McGinity Margaret (2008), “Currículum monocultural en la construcción de la identidad sociocultural de alumnos/ as de ascendencia mapuche”, en Organización y Gestión Educativa, N ${ }^{\circ}$ LXXII, año XVI, vol. $N^{\circ}$, pp. 34-35.

Rocher, Guy (1992), Introduction à la sociologie générale: regard sur la réalité sociale. Éditions Hurtubise, Montréal.

Rojas, Mónica y Sepúlveda, Segundo (2002), “Prejuicio étnico respecto de profesores mapuches de la ciudad de Temuco”. Tesis de Licenciatura en Educación. Facultad de Educación. Universidad Católica de Temuco, Temuco.

Ruiz Olabuenaga, José (1996), Metodología de la investigación cualitativa. Ediciones: Universidad de Deusto. Bilbao: España.

Sabourin, Paul (1997), "Perspective sur la mémoire sociale de Maurice Halbwachs”, en Sociologie et sociétés, vol. XXIX, $n^{\circ}$ 2, automne 1997, $p$. 139-161.

Saiz, José Luís, Merino, María, y Quilaqueo, Daniel (2009), “Meta-estereotipos sobre los indígenas mapuches de Chile”, en Interdisciplinaria, 26(1), 23-48.

Schnapper, Dominique (1998), La relation à l'autre. Au cœur de la pensée sociologique. Edition Gallimard, Paris.

Strauss, Anselm y Corbin, Juliette (2002), Bases de la investigación cualitativa. Técnicas y procedimientos para desarrollar la teoría fundamentada. Ediciones: Universidad de Antioquia. Medellín, Colombia.

Todorov, Tzvetan (1993), Las morales de la historia. Ediciones Paidós Ibérica S. A., Barcelona, España.

Trinidad, Antonio; Carrero, Virginia y Soriano, Rosa (2006), La construcción de la teoría a través del análisis interpretacional. Centro de Investigaciones Sociológicas, Madrid. 
Valles, Miguel (1997), Técnicas cualitativas de investigación social: Reflexión metodológica y práctica profesional. Síntesis, Madrid.

Van Dijk, Teum (1984), Prejudice in Discourse. An Analysis of Ethnic Prejudice in Cognition and Conversation. John Benjamin's Publishing Company, Amsterdam/Philadelphia.

Van Haecht, Anne (1999), La escuela va a examen. Preguntas a la sociología de la educación. Miño y Dávila Editores, Buenos Aires.

Vasquez, Ana \& Martinez, Isabel (1996), La socialisation à l'école: approche ethnographique. Presses Universitaires de France, Paris.

Wieviorka, Michel (1996), Une société fragmentée? Le multiculturalisme en débat. La Découverte, Paris.

Wodak, Ruth (2003), “De qué trata el análisis crítico del discurso (ACD). Resumen de su historia, sus conceptos fundamentales y sus desarrollos”, en Wodak, Ruth y Meyer, Mmichel, compiladores. (2003). Métodos de análisis crítico del discurso. Editorial Gedisa, Barcelona, pp. 17-34. 